\title{
Outcomes of Conservative Management of Splenic Injury Incurred During Percutaneous Nephrolithotomy
}

\author{
Vivian Paredes-Bhushan, MS, ${ }^{1}$ Eric P. Raffin, MD, ${ }^{2}$ John D. Denstedt, MD, ${ }^{3}$ Ben H. Chew, MD, ${ }^{4,5}$ \\ Bodo E. Knudsen, MD, ${ }^{5,6}$ Nicole L. Miller, MD, ${ }^{5,7}$ Manoj Monga, MD, 5,8 \\ Mark J. Noble, MD, ${ }^{8}$ and Vernon M. Pais, Jr., MD, MS ${ }^{2,5}$
}

\begin{abstract}
Purpose: Splenic injury is a rare complication after left-sided percutaneous nephrolithotomy (PCNL). Although initial observation is often espoused, the natural history of nonoperative conservative management is not well established and the implications of splenic injury are not fully defined in this context. We sought to describe outcomes of conservative management of splenic injury incurred at PCNL.

Patients and Methods: We performed a multi-institutional retrospective review of individual patients who underwent PCNL complicated by trans-splenic nephrostomy access injury. Demographic info, intraoperative data, management strategies, and outcomes were reviewed.

Results: Nine patients suffered splenic injury after left PCNL. All patients had supracostal upper pole access under fluoroscopic guidance. Splenic injury was identified by computed tomography (CT) in the eight of nine (89\%) who had imaging on first postoperative day. All eight patients were managed conservatively with nephrostomy dwell time of 2-21 days, one of whom (11\%) required blood transfusion. The remaining patient (11\%) - who had tubeless PCNL without postoperative imaging presented 5 days postoperatively with a delayed bleed and underwent emergent splenectomy. Seven of the nine (78\%) were managed nonoperatively and without need for transfusion or embolization.

Conclusion: The majority of patients incurring splenic injury during PCNL can be managed conservatively with maintenance of nephrostomy tube for $\geq 2$ days. Consequences of unrecognized splenic injury may include splenic bleed and may prompt transfusion and/or splenectomy, underscoring role of routine postoperative CT to allow timely diagnosis, particularly in those undergoing upper pole supracostal left-sided percutaneous renal access.
\end{abstract}

Keywords: percutaneous nephrolithotomy, splenic injury, conservative management

\section{Introduction}

$\mathbf{F}$ IRST ESTABLISHED IN the mid-1970s, percutaneous nephrolithotomy (PCNL) has become the preferred technique for removal of renal calculi that are $>2 \mathrm{~cm}$ or staghorn in physical configuration. PCNL can also be used for large stones when extracorporeal shock-wave lithotripsy or ureteroscopy are unsuccessful. In contrast to these interventions, PCNL demonstrates higher stone-free rate of $>90 \%$ and low retreatment rates. ${ }^{1-3}$ However, complications can occur from percutaneous renal access, including bleeding, transient fever, septicemia, extravasation of urine, and injury to adjacent structures. ${ }^{4}$

It has been advocated that supracostal access is preferable in certain situations, including in the extraction of staghorn calculi, superior caliceal, and ureteral stones. However, the risk of injury to adjacent structures may also be increased. ${ }^{5-8}$ This has been associated with iatrogenic complications,

\footnotetext{
${ }^{1}$ Dartmouth Geisel School of Medicine, Hanover, New Hampshire, USA.

${ }^{2}$ Section of Urology, Department of Surgery, Dartmouth Hitchcock Medical Center, Lebanon, New Hampshire, USA.

${ }^{3}$ Division of Urology, St. Joseph's Hospital, Western University, London, Canada.

${ }_{5}^{4}$ Department of Urologic Sciences, University of British Columbia, Vancouver, Canada.

${ }^{5}$ Endourology Disease Group for Excellence (EDGE) Research Consortium.

${ }^{6}$ Department of Urology, Ohio State University Medical Center, Columbus, Ohio, USA.

${ }^{7}$ Department of Urologic Surgery, Vanderbilt University Medical Center, Nashville, Tennessee, USA.

${ }^{8}$ Cleveland Clinic Glickman Urological and Kidney Institute, Cleveland, Ohio, USA.
} 
including injuries to the pleura, lung, liver, and colon. ${ }^{8,9}$ Although these complications are well documented, few have been published on splenic injuries.

To date, only 13 occurrences of splenic injury during PCNL have been reported in the literature. ${ }^{9-17}$ The largest previous series included only three patients. ${ }^{13}$ Although initial observation is often recommended, the outcomes of nonoperative/conservative management have not been well described. Furthermore, the short-term implications of splenic injury are not fully defined in the context of PCNL. Herein, we seek to describe the outcomes of conservative management of splenic injuries incurred with PCNL.

\section{Patients and Methods}

Upon approval by the Institutional Review Board, records of those undergoing PCNL were reviewed for occurrence of splenic injury from trans-splenic nephrostomy access at each of the participating medical centers. General demographic, perioperative, and postoperative outcome measures were collected, including timing at which the injuries were first identified, imaging modality used to identify the injury, nephrostomy access method and level, stone characteristics, length of hospital stay, subsequent duration of percutaneous renal drainage, estimated blood loss (EBL), and postoperative hemoglobin (Hgb) levels.

\section{Results}

There were a total of nine patients with splenic injury post-left PCNL. Sixty-seven percent were men $(n=6)$ and $33 \%$ were women $(n=3)$. The mean age at presentation was 48 years. The median body mass index was $28.2 \mathrm{~kg} / \mathrm{m}^{2}$ (range 24.8-47.4). One patient (11\%) had lymphoma. None of the patients had splenomegaly. The median EBL was $87.5 \mathrm{~mL}$ (range 75-200). The median $\mathrm{Hgb}$ level at the postanesthesia care unit (PACU) was $12.5 \mathrm{~g} / \mathrm{dL}$ (range 9.6-15.9). The median $\mathrm{Hgb}$ at postoperative day 1 (POD1) was $11.5 \mathrm{~g} / \mathrm{dL}$ (range 6.0-14.4). The median change in Hgb level from PACU and POD1 was $1.7 \mathrm{~g} / \mathrm{dL}$ (range $0.5-6.3$ ). The median largest stone size was $44.5 \mathrm{~mm}$ (range 12-91).

All patients had a preoperative computed tomography (CT) scan, none of which were preoperatively interpreted to demonstrate unusual splenic position. All patients had supracostal upper pole access under fluoroscopic guidance. Percutaneous access was achieved with the bulls-eye technique in five out of nine patients $(55.6 \%)$. The triangulation technique was used in the remaining four patients. Ultrasound guidance was not used for renal access. In all but one of the cases, access was obtained by the urologist. Radiology obtained fluoroscopic access with triangulation in the remaining patient. For one patient $(11.1 \%)$, nephrostomy access was made above the 10 th rib (Fig. 1). Access was made between 10th and 11th ribs for three patients $(33.3 \%)$. Access was made between the 11th and 12th ribs for five patients $(55.6 \%)$. Tracts were balloon dilated to 30F.

None of the injuries were identified intraoperatively. Transexamic acid was not used in any of the cases. All eight patients whose injuries were identified on postoperative CT underwent planned conservative management (Fig. 1). In these patients, the nephrostomy tube was not immediately

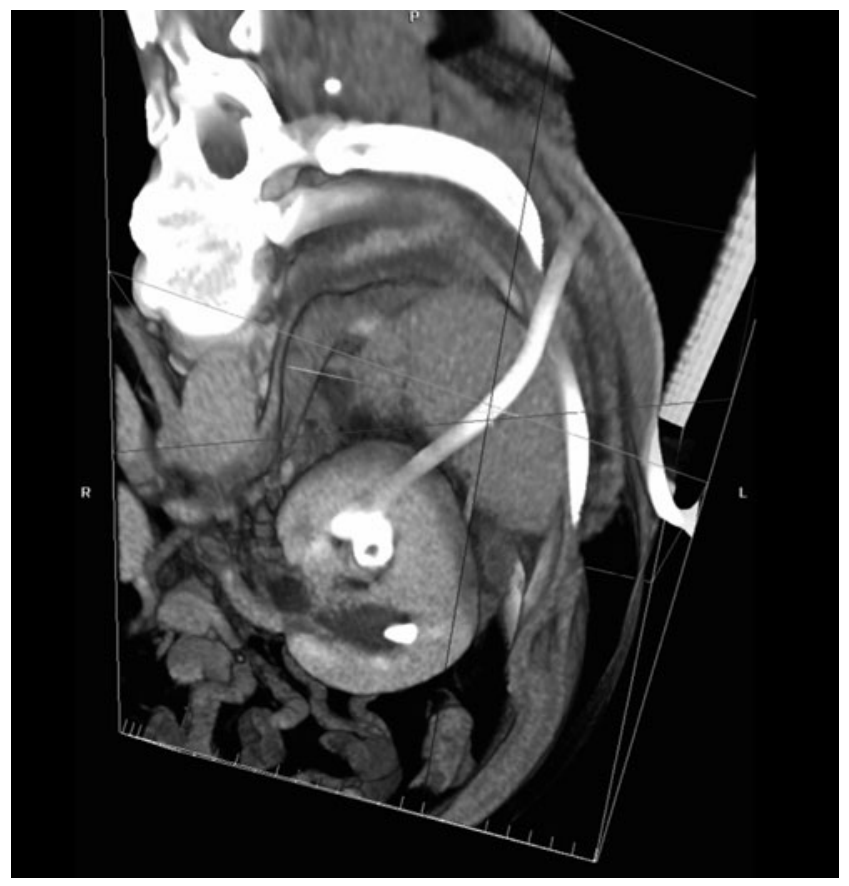

FIG. 1. A postoperative three-dimensional CT scan showing splenic injury after supracostal access above the 10th rib during left-sided PCNL. CT, computed tomography; PCNL, percutaneous nephrolithotomy.

removed, with a median nephrostomy dwell time of 6 days (range 2-21). The median length of stay was 4.5 days (range 1-7).

The one case in which access was obtained by a radiologist required transfusion with $6 \mathrm{U}$ of packed red blood cells perioperatively, with CT on the second POD revealing splenic injury with two nephrostomy tubes through the body of the spleen. The patient was managed nonoperatively, and both nephrostomy tubes were left in place for 5 days before removal. Collagen-thrombin hemostatic sealant (D-Stat; Vascular Solutions, Inc., Minneapolis, MN) was instilled through the two nephrostomy tracts at the time of removal, with angioembolization on standby. A CT angiography after nephrostomy tube removal showed no active bleeding, thus splenic embolization was not performed.

The patient who underwent tubeless PCNL had a $9 \mathrm{~cm}$ complete staghorn stone and had three tracts placed at the time of the procedure, including upper, inter, and lower pole. A ureteral stent was placed at the time of the procedure. She was clinically stable on POD1, her Hgb was $11.5 \mathrm{~g} / \mathrm{dL}$, and she was discharged. On POD5 she began to feel unwell at home and became dizzy and light-headed. An ambulance was called and she was transferred back to the medical center and assessed by the acute care surgical service. A CT scan indicated a splenic bleed (Fig. 2). Her Hgb at the time was $6.8 \mathrm{~g} / \mathrm{dL}$ and she was subsequently transfused with packed red blood cells, platelets, and fresh frozen plasma. She was taken to the operating room for an emergent splenectomy and stabilized immediately after surgery.

Overall, seven of nine patients $(77.8 \%)$ with splenic injury were managed conservatively without blood transfusion or 


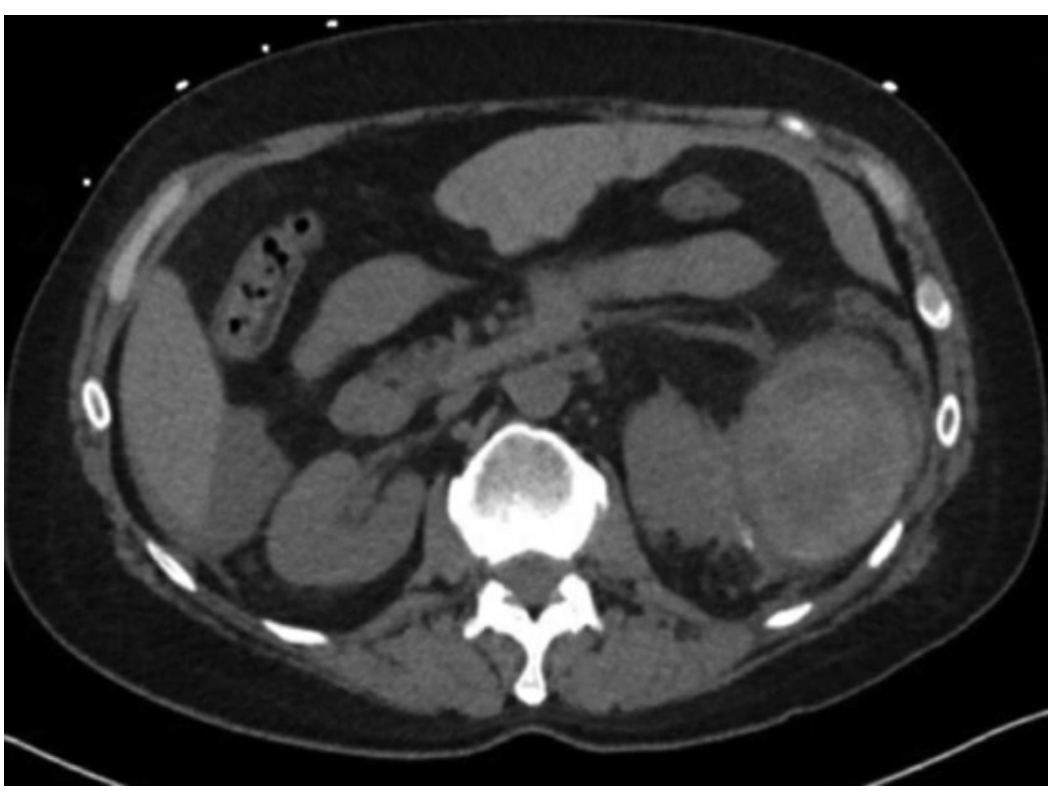

FIG. 2. A postoperative axial CT scan showing splenic injury 5 days after tubeless PCNL.

splenectomy. No patients underwent angioembolization. None had concurrent injuries. None had further sequalae or delayed bleed.

\section{Discussion}

Splenic injuries are an extremely rare complication of leftsided PCNL with few reported cases in the literature. ${ }^{10-12,14,15}$ Given the vascularity of the spleen and its proximity to the left kidney, it is prone to iatrogenic injury and subsequent hemorrhage during PCNL. ${ }^{4}$ In our series, eight patients had splenic injuries identified after the first POD through CT scan and were subsequently managed conservatively with close hemodynamic monitoring and $\mathrm{Hgb}$ assessment.

The level of percutaneous access may result in increased risk of splenic injury. Hopper and Yakes demonstrated that the potential rate of splenic injury is roughly $33 \%$ with a prone intercostal approach between the 10th and 11th ribs under full expiration. ${ }^{6}$ Similarly, Shah et al. also demonstrated an increased risk of splenic injury with a 10th intercostal approach. ${ }^{6,12}$ Although it has been shown that there is minimal risk in puncturing the spleen with a posterior 11th12 th rib intercostal approach at full expiration, more than half of our patients were accessed between the 11th and 12th ribs. Although this was not specifically assessed in our study, stones with increased complexity may require multiple PCNL tracts for retrieval, which in turn can increase the risk of iatrogenic injury.

Subclinical or occult splenic injuries are likely to go underreported and unrecognized since CT imaging is not routinely used at all centers in the postoperative management of patients undergoing PCNL. ${ }^{14}$ Although CT is useful for identifying residual stone burden, ${ }^{16,18,19}$ it has also been shown to be useful in identifying postoperative complications, including splenic injuries. ${ }^{20}$ It can assess for intraabdominal hemorrhages as well as classifying and grading severity of splenic injuries. ${ }^{21-23}$ In our case series, all cases of conservative management of splenic injuries after PCNL occurred in patients who underwent CT imaging within the first or second POD. With a sensitivity and specificity of $96 \%$ in identifying these complications, ${ }^{24}$ postoperative CT imaging should be considered in patients undergoing upper pole, supracostal left PCNL, particularly if preoperative imaging suggests proximity of spleen to percutaneous renal access.

Fluoroscopic guidance is the most commonly used access technique for percutaneous renal access in the United States and all of our patients had fluoroscopic access. Ultrasound guidance is an alternative for gaining percutaneous renal access and can be an attractive option because of its low cost and ability to view organs within the tract. Despite current underutilization in the United States, ultrasonography is increasingly embraced for renal percutaneous access in PCNL. Ultrasonography provides real-time three dimensional observation of the kidney in addition to other neighboring structures. ${ }^{25,26}$ Additional advantages of ultrasound guidance over fluoroscopy in PCNL include lack of radiation exposure, and lack of exposure to contrast media. It can also be safely utilized to acquire access in supine PCNL. Agarwal et al. evaluated the use of ultrasound as an adjunct to fluoroscopy in obtaining renal percutaneous access, demonstrating significant reductions in mean times for effective puncture, and duration of radiation exposure, with a combination of fluoroscopy and ultrasound compared with those accessed fluoroscopically without ultrasound. ${ }^{26}$ Ultrasound guidance has suggested limitations, including purported reduced precision in comparison with fluoroscopy, with particular challenges in obese patients. ${ }^{25}$ Nevertheless, use of ultrasound has been described as an important factor in preventing PCNL-related complications, which also include injury to the spleen. ${ }^{4} \mathrm{Al}-$ though it is conceivable that intraoperative ultrasound may have reduced the likelihood of splenic injury in this cohort, case series design does not allow assessment of this question.

Splenic injuries incurred from trauma may be managed conservatively and operatively. Nonoperative management is increasing in popularity among both blunt and penetrating splenic trauma with the advent of imaging and newer trauma grading systems. ${ }^{22,23,27}$ Initially presumed to be correlated 
with high failure rates of nonoperative management, patients with high-grade splenic injuries can be treated by conservative management provided they are hemodynamically stable. Early elective angioembolization has also been shown to increase the success rates of nonoperative management among individuals with high-grade splenic injuries. ${ }^{27,28}$ In the context of splenic injuries incurred on PCNL, conservative management may entail bed rest, close hemodynamic and $\mathrm{Hgb}$ or hematocrit monitoring, and extended maintenance of nephrostomy catheter in nephrostomy tracts, which can aid in avoiding further morbidity and mortality from splenectomy. ${ }^{14}$ Collagen-thrombin hemostatic sealants have also been described in the treatment of splenic injuries post-PCNL. ${ }^{12,14,15}$ Thomas et al. described a patient with splenic injury identified post-PCNL treated with D-Stat after removal of nephrostomy tube. ${ }^{14}$ Similarly, Desai et al. described an injury that was managed conservatively only with an absorbable gelatin powder $\left(\right.$ Gelfoam $^{\circledR}$; Pfizer, New York, NY). ${ }^{15}$ Apart from one patient who was treated with D-Stat injected through the tracts upon nephrostomy tube removal, conservative management did not require treatment with a hemostatic sealant for other patients. Extrapolating from The Eastern Association for the Surgery of Trauma practice management guideline, it is important to note that candidates for nonoperative management must be hemodynamically stable with isolated splenic injuries. Furthermore, age $>55$ years, concomitant injuries, neurologic status, and severity of splenic trauma are not contraindications for nonoperative management, provided that the patient is hemodynamically stable. ${ }^{4,29,30}$ Hemodynamically unstable patients secondary to severe hemorrhage from penetrating trauma may require emergent exploratory laparotomy for splenic repair or splenectomy. ${ }^{4,29}$

We present the largest case series of post-PCNL splenic injury to date; however, several limitations must be acknowledged. Our small sample size limits generalizability. In addition, the retrospective nature of this study increases the risk of selection bias and confounding. The absence of a standardized protocol for postoperative removal of nephrostomy tubes contributed to the heterogeneity of management seen in our cohort. Currently, there is no defined minimal indwell time for a nephrostomy tube after PCNLrelated splenic injury. Various reports in the literature have described duration ranging from 4 days up to a little $>2$ weeks. ${ }^{9,11,13,16,17}$ Despite these limitations, our series assesses a rare complication of left-sided PCNL and highlights that postoperative CT imaging in the subset of patients undergoing left renal upper pole access may be useful in the diagnosis of splenic injuries. Effective conservative management with early postoperative recognition is demonstrated.

\section{Conclusion}

The majority of patients with splenic injury sustained from PCNL can be managed nonoperatively. Despite the rarity of this complication, in patients undergoing upper pole supracostal left-sided percutaneous renal access, clinicians should have an elevated index of suspicion for possibility of splenic transgression and consider CT imaging as clinically indicated. In the absence of postoperative CT-identified splenic injury, delayed bleeding may occur, thus clinicians should remain vigilant in those with supracostal left PCNL during their postoperative recovery and counsel patients accordingly.

\section{Author Disclosure Statement}

No competing financial interests exist.

\section{Funding Information}

No funding was received for this article.

\section{References}

1. Lahme S, Bichler K-H, Strohmaier WL, Götz T. Minimally invasive PCNL in patients with renal pelvic and calyceal stones. Eur Urol 2001;40:619-624.

2. Shokeir AA, Sheir KZ, El-Nahas AR, El-Assmy AM, Eassa W, El-Kappany HA. Treatment of renal stones in children: A comparison between percutaneous nephrolithotomy and shock wave lithotripsy. J Urol 2006;176:706-710.

3. Akman T, Binbay M, Ozgor F, et al. Comparison of percutaneous nephrolithotomy and retrograde flexible nephrolithotripsy for the management of $2-4 \mathrm{~cm}$ stones: A matched-pair analysis. BJU Int 2012;109:1384-1389.

4. Michel MS, Trojan L, Rassweiler JJ. Complications in percutaneous nephrolithotomy. Eur Urol 2007;51:899_ 906.

5. Hopper KD, Chantelois AE. The retrorenal spleen. Implications for percutaneous left renal invasive procedures. Invest Radiol 1989;24:592-595.

6. Hopper K, Yakes W. The posterior intercostal approach for percutaneous renal procedures: Risk of puncturing the lung, spleen, and liver as determined by CT. AJR Am J Roentgenol 1990;154:115-117.

7. Gupta R, Kumar A, Kapoor R, Srivastava A, Mandhani A. Prospective evaluation of safety and efficacy of the supracostal approach for percutaneous nephrolithotomy. BJU Int 2002;90:809-813.

8. Öztürk H. Gastrointestinal system complications in percutaneous nephrolithotomy: A systematic review. J Endourol 2014;28:1256-1267.

9. Gaunay GS, Ahmed H, Smith A, Okeke Z. Conservative management of combined pleural and splenic injury during percutaneous nephrostolithotomy. J Endourol Case Rep 2016;2:176-179.

10. Kondas J, Szentgyörgyi E, Vaczi L, Kiss A. Splenic injury: A rare complication of percutaneous nephrolithotomy. Int Urol Nephrol 1994;26:399-404.

11. Carey RI, Siddiq FM, Guerra J, Bird VG. Conservative management of a splenic injury related to percutaneous nephrostolithotomy. JSLS 2006;10:504.

12. Shah HN, Hegde SS, Mahajan AP, Sodha H, Shah R, Bansal M. Splenic injury: Rare complication of percutaneous nephrolithotomy: Report of two cases with review of literature. J Endourol 2007;21:919-922.

13. Schaeffer AJ, Handa SE, Lingeman JE, Matlaga BR. Transsplenic percutaneous nephrolithotomy. J Endourol 2008;22:2481-2484.

14. Thomas AA, Pierce G, Walsh RM, Sands M, Noble M. Splenic injury during percutaneous nephrolithotomy. JSLS 2009;13:233.

15. Desai AC, Jain S, Benway BM, Grubb RL, III, Picus D, Figenshau RS. Splenic injury during percutaneous nephrolithotomy: A case report with novel management technique. J Endourol 2010;24:541-545. 
16. Gnessin E, Mandeville JA, Handa SE, Lingeman JE. The utility of noncontrast computed tomography in the prompt diagnosis of postoperative complications after percutaneous nephrolithotomy. J Endourol 2012;26:347-350.

17. Ozlu D, Seker K, Sam E, Atar FA. Splenic injury during percutaneous nephrolithotomy: A case report of a rare complication. Cureus 2019;11:e6298.

18. Osman Y, El-Tabey N, Refai H, et al. Detection of residual stones after percutaneous nephrolithotomy: Role of nonenhanced spiral computerized tomography. J Urol 2008;179: 198-200.

19. Bee TK, Croce MA, Miller PR, Pritchard FE, Fabian TC. Failures of splenic nonoperative management: Is the glass half empty or half full? J Trauma Acute Care Surg 2001;50: 230-236.

20. Semins MJ, Bartik L, Chew BH, et al. Multicenter analysis of postoperative CT findings after percutaneous nephrolithotomy: Defining complication rates. Urology 2011; 78:291-294.

21. Demetriades D, Hadjizacharia P, Constantinou C, et al. Selective nonoperative management of penetrating $a b-$ dominal solid organ injuries. Ann Surg 2006;244:620.

22. Kozar RA, Crandall M, Shanmuganathan K, et al. Organ injury scaling 2018 update: Spleen, liver, and kidney. J Trauma Acute Care Surg 2018;85:1119-1122.

23. Moore EE, Cogbill TH, Jurkovich GJ, Shackford SR, Malangoni MA, Champion HR. Organ injury scaling: Spleen and liver (1994 revision). J Trauma Acute Care Surg 1995; 38:323-324.

24. Robertson F, Leander P, Ekberg O. Radiology of the spleen. Eur Radiol 2001;11:80-95.

25. Chu C, Masic S, Usawachintachit M, et al. Ultrasoundguided renal access for percutaneous nephrolithotomy: A description of three novel ultrasound-guided needle techniques. J Endourol 2016;30:153-158.

26. Agarwal M, Agrawal MS, Jaiswal A, Kumar D, Yadav H, Lavania P. Safety and efficacy of ultrasonography as an adjunct to fluoroscopy for renal access in percutaneous nephrolithotomy (PCNL). BJU Int 2011;108:1346-1349.

27. Knudson MM, Maull KI. Nonoperative management of solid organ injuries: Past, present, and future. Surg Clin 1999;79:1357-1371.

28. Dent D, Alsabrook G, Erickson BA, et al. Blunt splenic injuries: High nonoperative management rate can be achieved with selective embolization. J Trauma Acute Care Surg 2004; 56:1063-1067.

29. Berg RJ, Inaba K, Okoye O, et al. The contemporary management of penetrating splenic injury. Injury 2014;45: 1394-1400.

30. Stassen NA, Bhullar I, Cheng JD, et al. Selective nonoperative management of blunt splenic injury: An Eastern Association for the Surgery of Trauma practice management guideline. J Trauma Acute Care Surg 2012;73:S294 S300.

Address correspondence to: Vivian Paredes-Bhushan, MS Dartmouth Geisel School of Medicine

One Rope Ferry Road Hanover, NH 03755

USA

E-mail: vivian.paredes.bhushan.med@dartmouth.edu

$$
\begin{aligned}
& \text { Abbreviations Used } \\
\mathrm{CT} & =\text { computed tomography } \\
\mathrm{EBL} & =\text { estimated blood loss } \\
\mathrm{Hgb} & =\text { hemoglobin } \\
\mathrm{PACU} & =\text { postanesthesia care unit } \\
\mathrm{PCNL} & =\text { percutaneous nephrolithotomy } \\
\mathrm{POD} 1 & =\text { postoperative day } 1
\end{aligned}
$$

